\title{
Are the gyro-ages of field stars underestimated? (Corrigendum)
}

Géza Kovács

Konkoly Observatory, Budapest, Hungary e-mail: kovacs@konkoly.hu

A\&A, 581, A2 (2015) https://doi .org/10.1051/0004-6361/201525920

Key words. stars: rotation - starspots - open clusters and associations: general - errata, addenda

Due to an unfortunate typo, the statement about the ages of the Galactic field stars is just the opposite of what is given in the Abstract. The correct statement is the following: "We show that, in the overall sense, the isochrone ages are systematically greater than the gyro-ages". The rest of the paper is in agreement with this statement. 